\title{
Hydrostatic grounding line parameterization in ice sheet models
}

\author{
H. Seroussi ${ }^{1}$, M. Morlighem ${ }^{2}$, E. Larour ${ }^{1}$, E. Rignot $^{2,1}$, and A. Khazendar ${ }^{1}$ \\ ${ }^{1}$ Jet Propulsion Laboratory, California Institute of Technology, 4800 Oak Grove Drive MS 300-323, \\ Pasadena, CA 91109-8099, USA \\ ${ }^{2}$ University of California, Irvine, Department of Earth System Science, Croul Hall, Irvine, CA 92697-3100, USA \\ Correspondence to: H. Seroussi (helene.seroussi@jpl.nasa.gov)
}

Received: 3 June 2014 - Published in The Cryosphere Discuss.: 24 June 2014

Revised: 6 October 2014 - Accepted: 8 October 2014 - Published: 17 November 2014

\begin{abstract}
Modeling of grounding line migration is essential to accurately simulate the behavior of marine ice sheets and investigate their stability. Here, we assess the sensitivity of numerical models to the parameterization of the grounding line position. We run the MISMIP3D benchmark experiments using the Ice Sheet System Model (ISSM) and a two-dimensional shelfy-stream approximation (SSA) model with different mesh resolutions and different sub-element parameterizations of grounding line position. Results show that different grounding line parameterizations lead to different steady state grounding line positions as well as different retreat/advance rates. Our simulations explain why some vertically depth-averaged model simulations deviate significantly from the vast majority of simulations based on SSA in the MISMIP3D benchmark. The results reveal that differences between simulations performed with and without sub-element parameterization are as large as those performed with different approximations of the stress balance equations in this configuration. They also demonstrate that the reversibility test is passed at relatively coarse resolution while much finer resolutions are needed to accurately capture the steady-state grounding line position. We conclude that fixed grid SSA models that do not employ such a parameterization should be avoided, as they do not provide accurate estimates of grounding line dynamics, even at high spatial resolution. For models that include sub-element grounding line parameterization, in the MISMIP3D configuration, a mesh resolution finer than $2 \mathrm{~km}$ should be employed.
\end{abstract}

\section{Introduction}

Mapping of grounding lines, where ice detaches from the underlying bedrock and becomes afloat in the ocean, is possible using satellite remote sensing with either visible imagery (Bohlander and Scambos, 2007) or differential radar interferometry (Goldstein et al., 1993; Rignot et al., 2011b). Observations show that grounding lines have a dynamic behavior. This is particularly the case in the Amundsen Sea sector of West Antarctica, where their migration inland reaches more than $1 \mathrm{~km} \mathrm{yr}^{-1}$ on Pine Island and Thwaites Glacier (Rignot et al., 2011a). Accurate knowledge of grounding line positions as well as their evolution in time is therefore critical to understand ice sheet dynamics. Grounding lines are indeed a fundamental control of marine ice sheet stability (van der Veen, 1985; Hindmarsh and Le Meur, 2001), and they also determine the shape of ice-shelf cavities, which affect oceaninduced melting rates (Schodlok et al., 2012). Grounding line dynamics are strongly non-linear, with long episodes of relative stability interrupted by significant retreat, this evolution being controlled, among other factors, by basal topography (Weertman, 1974; Durand et al., 2009b). The Antarctic ice sheet is surrounded by floating ice shelves of varying size, and modeling of this transition zone is therefore essential to simulate the evolution of polar ice sheets in our changing climate.

However, accurate modeling of this transition zone remains both a scientific and technical challenge. Threedimensional (3-D) full-Stokes (FS) models are required in order to fully resolve the contact problem between the ice and the underlying bedrock (Nowicki and Wingham, 2008; Durand et al., 2009b, a; Favier et al., 2014). This approach is computationally intensive and sensitive to model data, so it 
has been applied to synthetic geometries mainly and starts to be applied for real glaciers (Favier et al., 2014). Alternative approaches that have been widely used rely on the hydrostatic criterion to estimate the grounding line position: ice shelves are assumed to float hydrostatically in ocean water (Huybrechts, 1990; van der Veen, 1985; Ritz et al., 2001). Models often rely on fixed grids or meshes for which each grid cell or element is either entirely floating or entirely grounded. This method limits the precision of the grounding line position and simulations show a strong dependency on grid size. A fine mesh resolution is then required in the grounding zone in order to accurately capture grounding line migration and reduce numerical artifacts caused by model discretization (Vieli and Payne, 2005; Katz and Worster, 2010). Using sub-grid parameterization, which tracks the grounding line position within the element, improves models based on hydrostatic equilibrium condition and reduces their dependency on grid size (Pattyn et al., 2006; Gladstone et al., 2010a; Winkelmann et al., 2011). Another alternative is to use moving grid or adaptive mesh refinement, so that the mesh or grid resolution follows the grounding line transition zone (Goldberg et al., 2009; Cornford et al., 2013). These methods overcome the difficulties associated to grounding line discretization but lead to more complicated frameworks and remain difficult to implement in parallelized architectures. Due to the high computational time associated with fine resolution meshes or grids, most studies investigating the impact of grounding line parameterization, mesh resolution or stress balance approximation are performed on onedimensional (1-D) flow line or two-dimensional (2-D) flowband models (e.g., Vieli and Payne, 2005; Pattyn et al., 2006; Schoof, 2007a, b; Katz and Worster, 2010; Gladstone et al., 2010a; Pattyn et al., 2012). They show the strong dependency of model results on mesh resolution in the grounding line transition zone. They also demonstrate that moving grid models explicitly tracking grounding line position are able to reduce the dependency of results on mesh resolution. Analyses on 2-D plan-view or 3-D models confirm these results (Goldberg et al., 2009; Cornford et al., 2013). Recent results using plan-view shallow models and finite differences (Feldmann et al., 2014) also show that including grounding line sub-grid parameterization in shallow models allows to capture grounding line reversibility at low resolutions without including a flux correction.

Benchmark efforts, such as the Marine Ice Sheet Model Intercomparison Project (MISMIP), that compare results from a variety of ice flow models and spatial resolutions, have been performed for both flow line (MISMIP) and plan-view (MISMIP3D) models. They compare the sensitivity of modeled grounding line migration to numerical implementation (Pattyn et al., 2012, 2013; Pattyn and Durand, 2013). Results indicate that plan-view models need to include at least membrane stress components to be able to capture the grounding line position and that this position depends on the degree of sophistication of the model. Results also emphasize the need to use spatial resolution finer than $500 \mathrm{~m}$ when relying on fixed grid discretization and finer than $5 \mathrm{~km}$ when sub-grid parameterizations are included in the MISMIP3D configurations (Pattyn et al., 2013).

These conclusions are however drawn from a variety of models based on different softwares and different approximations for the stress balance equations, with different grounding line parameterizations, using either structured or unstructured meshes that are either fixed or adapted with time. It is therefore difficult to attribute the differences of the model results to either the approximation made in the stress balance equations or to the parameterization adopted to capture the grounding line position. In the MISMIP3D experiments, for example, some results based on the shelfy-stream approximation (SSA, MacAyeal, 1989) deviate significantly from the vast majority of SSA model results. The differences in the grounding line positions between models based on the SSA are either due to differences in grounding line parameterization or domain discretization.

In this study we assess the impact of different sub-element parameterizations for hydrostatic grounding line treatment using a single ice flow model. Experiments are based on the MISMIP3D configurations. We use the Ice Sheet System Model (ISSM, Larour et al., 2012) to solve the 2-D shelfy-stream equations with spatial resolutions varying between $5 \mathrm{~km}$ and $250 \mathrm{~m}$. We analyze the grounding line steady state position, its evolution following a perturbation in basal friction and the reversibility of its evolution for the different grounding line parameterizations. We conclude on the requirements needed to accurately capture grounding line motion and the impact of the underlying parameterization.

\section{Model}

\subsection{Field equations}

The 2-D SSA is employed for both grounded and floating ice, so membrane stress terms are included but all vertical shearing is neglected. Ice viscosity, $\mu$, is considered to be isotropic and to follow Glen's flow law (Cuffey and Paterson, 2010):

$\mu=\frac{B}{2 \dot{\epsilon}^{\frac{n-1}{n}}}$,

where $B$ is the ice viscosity parameter, $\dot{\epsilon}_{\mathrm{e}}$ the effective strain rate and $n=3$ Glen's exponent.

A non-linear friction law that links basal shear stress to basal sliding velocity is applied on grounded ice:

$\boldsymbol{\tau}_{\mathrm{b}}=C\left|\boldsymbol{u}_{\mathrm{b}}\right|^{m-1} \boldsymbol{u}_{\mathrm{b}}$,

where $\boldsymbol{\tau}_{\mathrm{b}}$ is the basal shear stress, $\boldsymbol{u}_{\mathrm{b}}$ is the basal sliding velocity, $C$ is the friction coefficient, and $m$ is the sliding law exponent. $C$ is defined on each node and the friction coefficient therefore varies linearly within an element. Thickness evolution is dictated by mass conservation. 

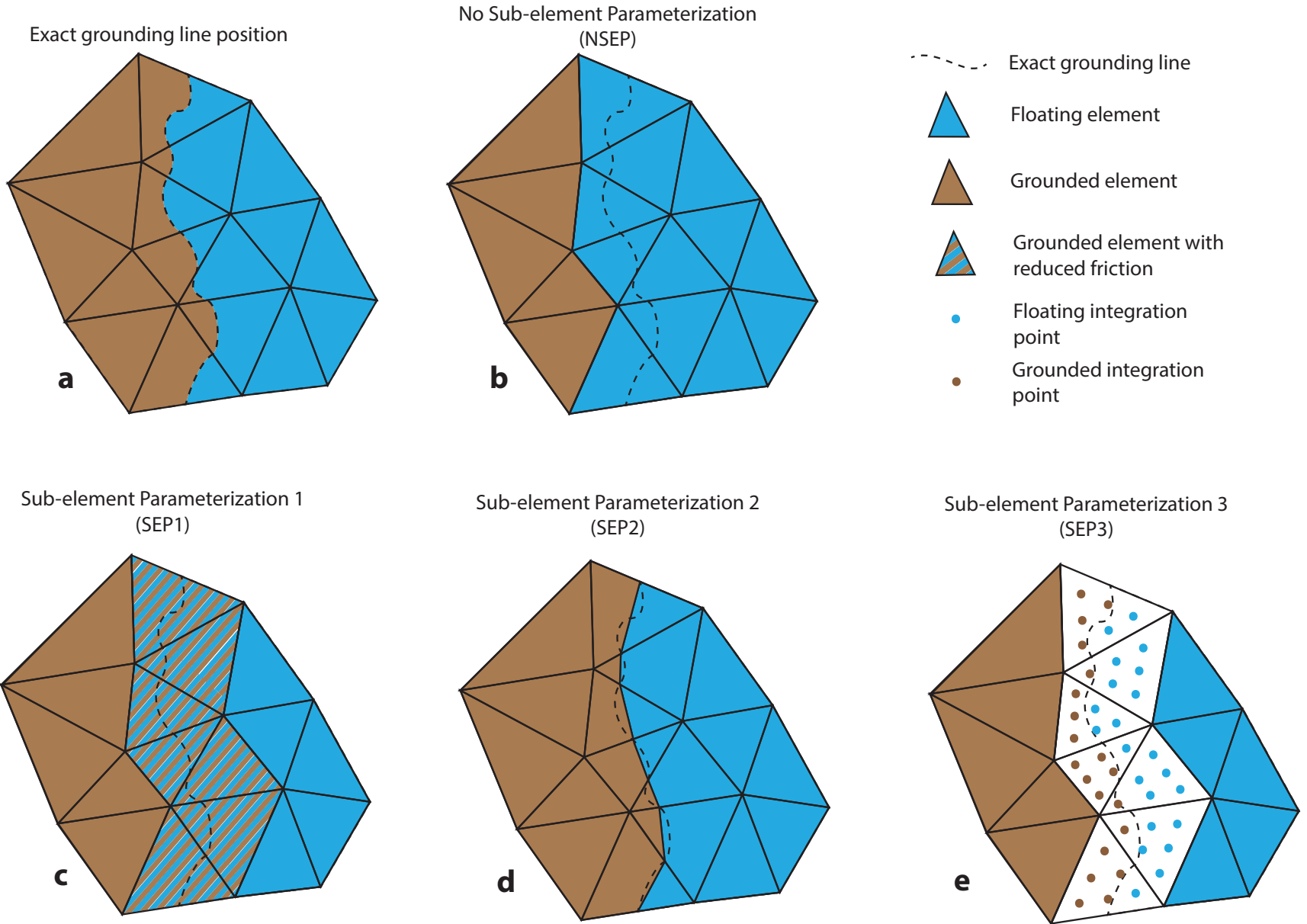

Figure 1. Grounding line discretization. Grounding line exact location (a), no sub-element parameterization (NSEP, b), sub-element parameterization 1 (SEP1, c), sub-element parameterization 2 (SEP2, d) and sub-element parameterization 3 (SEP3, e).

The position of the grounding line is determined by a floatation criterion: ice is floating if its thickness, $H$, is equal or lower than the floating height $H_{\mathrm{f}}$ defined as follows:

$H_{\mathrm{f}}=-\frac{\rho_{\mathrm{w}}}{\rho_{\mathrm{i}}} r, \quad r<0$,

where $\rho_{\mathrm{i}}$ is the ice density, $\rho_{\mathrm{W}}$ the ocean density and $r$ the bedrock elevation (negative if below sea level). Grounding line is therefore located where $H=H_{\mathrm{f}}$ :

$H>H_{\mathrm{f}}$ ice is grounded,

$H=H_{\mathrm{f}}$ grounding line position,

$H<H_{\mathrm{f}}$ ice is floating.

\subsection{Domain discretization}

The domain is discretized with a 2-D isotropic uniform unstructured triangle mesh. Velocity and geometry fields are computed on each vertex of the mesh using Lagrange P1 (piecewise linear) finite elements. Element size varies between $5 \mathrm{~km}$ for the lowest resolution and $250 \mathrm{~m}$ for the highest resolution and is uniform within each mesh.
Grounding line position (Fig. 1a) is based on the hydrostatic equilibrium condition as described above and three different techniques are used to parameterize its position. As the same SSA equations are used on the entire domain to compute the stress balance, the only difference between grounded and floating ice is the presence or absence of basal friction.

In the first method, each element of the mesh is either grounded or floating: floatation criterion is determined on each vertex of the triangle and if at least one vertex of the triangle is floating, the element is considered floating and no friction is applied. Otherwise, if the three vertices are grounded, the element is considered grounded. This is the simplest approach used by fixed grid models to determine grounding line positions (Vieli and Payne, 2005), in which the grounding line is defined as the last grounded point. We refer to this technique as no sub-element parameterization (NSEP, Fig. 1b).

In the second method, the floating condition is a 2-D field and the grounding line position is determined by the line where $H=H_{\mathrm{f}}$, so it is located anywhere within an element. 
Some elements are therefore partly grounded and partly floating. In this case the initial basal friction $C$ is reduced to match the amount of grounded ice in the element as proposed by Pattyn et al. (2006) and Gladstone et al. (2010a) but for a 2-D element:

$C_{\mathrm{g}}=C \frac{A_{\mathrm{g}}}{A}$,

where $C_{\mathrm{g}}$ is the applied basal friction coefficient for the element partially grounded, $A_{\mathrm{g}}$ is the area of grounded ice of this element and $A$ is the total area of the element. As all fields and data are computed using piecewise linear function, the grounding line position within each triangle is a straight line. This technique is referred to as sub-element parameterization 1 (SEP1, Fig. 1c) in the remainder of the paper.

In the third method, the grounding line position is located anywhere within an element as for SEP1, but the basal friction computed for partly grounded elements differs. We take advantage of finite element properties to integrate the basal friction only on the part of the element that is grounded. This can be done simply by changing the integration area from the initial element to the grounded part of the element, over which the basal friction is unchanged. This technique is referred to as sub-element parameterization 2 (SEP2, Fig. 1d) in the remainder of the manuscript.

In the fourth method, the sub-element parameterization is based on the number of integration points. We test the performance of this method by looking at the steady-state grounding line position (see experiments description below) for spatial resolutions of 1 and $5 \mathrm{~km}$. The finite element method consists of calculating integrals over each element using a given set of integration points, also called Gaussian quadrature (Zienkiewicz and Taylor, 1989). The number of integration points in each element depends on the degree of polynomial functions being integrated, with more integration points required for polynomial functions of higher degree. In our case, the basal friction goes from zero on the floating part of the element to the value specified in the experiment section, so this step function would require an infinite number of integration points to be exact. An alternative to the two SEP described above is to increase the number of integration points in the integrals and include basal friction for integration points whose thickness is higher than the floating height. SEP3 only allows a finite number of grounding line positions to be captured within the element contrary to the other two SEP. We tested this alternative solution on the 1 and $5 \mathrm{~km}$ meshes, with integration orders going from 2 to 20, which is equivalent to a number of integration points varying between 3 and 79. This technique is referred to as sub-element parameterization 3 (SEP3, Fig. 1e).

Appendix A details the different descriptions of the stiffness matrix associated to basal friction for all the subelement parameterizations.

\section{Experiments}

We reproduce the MISMIP3D setup (Pattyn et al., 2013) and run similar experiments to investigate the influence of spatial resolution and grounding line parameterization on grounding line position and migration. Ice flows over a bedrock with a constant downward sloping bed that varies only in the $x$ direction. The bedrock elevation is defined as follows:

$b(x, y)=-100-x$.

Ice viscosity parameter, $B$, is uniform over the whole domain and equal to $2.15 \times 10^{8} \mathrm{~Pa} \mathrm{~s}^{-1 / 3}$; the basal friction coefficient, $C$, is also uniform for all grounded ice and equal to $10^{7} \mathrm{~Pa} \mathrm{~m}^{-1 / 3} \mathrm{~s}^{1 / 3}$, so $C$ is constant over each element except for those containing the grounding line, where it varies linearly; the friction law exponent, $m$ is equal to $1 / 3$. The domain is rectangular and stretches between 0 and $800 \mathrm{~km}$ in the $x$ direction and 0 and $50 \mathrm{~km}$ in the $y$ direction. The boundary conditions applied are as follows: a symmetric ice divide is considered at $x=0$ so the velocity is equal to zero. Water pressure is applied at $x=800 \mathrm{~km}$ to model contact with the ocean. There is a symmetry axis at $y=0$ that represents the centerline of the ice stream and a free slip condition for $y=50 \mathrm{~km}$, so there is no flux advected through these surfaces and the tangential velocity is equal to zero.

Starting from a thin layer of ice of $10 \mathrm{~m}$, a constant accumulation $\dot{a}$ of $0.5 \mathrm{~m} \mathrm{yr}^{-1}$ is applied over the whole domain. The marine ice sheet evolves until a steady state configuration is reached. At each time step, we compute the ice velocity, its thickness, the new grounding line position and update the upper and lower surfaces.

This steady state configuration is then perturbed by changing the basal friction coefficient $C$. This parameter is adjusted spatially using a Gaussian bump such that

$C^{*}=C\left[1-0.75 \exp \left(-\frac{\left(x-x_{\mathrm{b}}\right)^{2}}{2 x_{\mathrm{c}}^{2}}-\frac{\left(y-y_{\mathrm{b}}\right)^{2}}{2 y_{\mathrm{c}}^{2}}\right)\right]$,

with $C^{*}$ as the new friction coefficient, $x_{\mathrm{b}}$ as the grounding line position at $y=0 \mathrm{~km}$ in the steady state configuration, $y_{\mathrm{b}}=0, x_{\mathrm{c}}=150 \mathrm{~km}$, and $y_{\mathrm{c}}=10 \mathrm{~km}$ as the spatial extent of the perturbation along the $x$ and $y$ directions. The model is run forward in time for 100 years. The sliding friction is then reset to its initial uniform value and the model runs forward in time until a new steady state configuration is reached. This experiment is designed to assess the ability of models to provide reversible grounding line positions under simplified conditions (Pattyn et al., 2013). The marine ice sheet theory states that ice resting on a down sloping bed without lateral variations exhibits only one steady state grounding line position (Schoof, 2007b). MISMIP benchmark demonstrated that failure to reproduce the reversibility test is often associated with coarse mesh resolution.

Steady state and reduced friction experiments are run with five different meshes, with spatial resolution ranging 
Table 1. Initial grounding line position and span for the NSEP, SEP1 and SEP2 simulations.

\begin{tabular}{llcrl}
\hline GL parameterization & Resolution & GL $(y=0 \mathrm{~km})$ & GL $(y=50 \mathrm{~km})$ & $\delta \mathrm{GL}$ \\
\hline NSEP & $5 \mathrm{~km}$ & $187.5 \mathrm{~km}$ & $188.4 \mathrm{~km}$ & $5009 \mathrm{~m}$ \\
NSEP & $2 \mathrm{~km}$ & $406.3 \mathrm{~km}$ & $407.6 \mathrm{~km}$ & $3439 \mathrm{~m}$ \\
NSEP & $1 \mathrm{~km}$ & $481.2 \mathrm{~km}$ & $480.3 \mathrm{~km}$ & $2000 \mathrm{~m}$ \\
NSEP & $500 \mathrm{~m}$ & $522.7 \mathrm{~km}$ & $522.2 \mathrm{~km}$ & $879 \mathrm{~m}$ \\
NSEP & $250 \mathrm{~m}$ & $558.4 \mathrm{~km}$ & $558.2 \mathrm{~km}$ & $440 \mathrm{~m}$ \\
\hline SEP1 & $5 \mathrm{~km}$ & $631.7 \mathrm{~km}$ & $631.9 \mathrm{~km}$ & $782 \mathrm{~m}$ \\
SEP1 & $2 \mathrm{~km}$ & $609.8 \mathrm{~km}$ & $610.2 \mathrm{~km}$ & $670 \mathrm{~m}$ \\
SEP1 & $1 \mathrm{~km}$ & $604.9 \mathrm{~km}$ & $604.8 \mathrm{~km}$ & $292 \mathrm{~m}$ \\
SEP1 & $500 \mathrm{~m}$ & $605.0 \mathrm{~km}$ & $605.0 \mathrm{~km}$ & $148 \mathrm{~m}$ \\
SEP1 & $250 \mathrm{~m}$ & $605.5 \mathrm{~km}$ & $605.6 \mathrm{~km}$ & $108 \mathrm{~m}$ \\
\hline SEP2 & $5 \mathrm{~km}$ & $550.3 \mathrm{~km}$ & $551.1 \mathrm{~km}$ & $1215 \mathrm{~m}$ \\
SEP2 & $2 \mathrm{~km}$ & $575.0 \mathrm{~km}$ & $574.8 \mathrm{~km}$ & $429 \mathrm{~m}$ \\
SEP2 & $1 \mathrm{~km}$ & $592.2 \mathrm{~km}$ & $591.9 \mathrm{~km}$ & $381 \mathrm{~m}$ \\
SEP2 & $500 \mathrm{~m}$ & $599.1 \mathrm{~km}$ & $599.1 \mathrm{~km}$ & $170 \mathrm{~m}$ \\
SEP2 & $250 \mathrm{~m}$ & $603.3 \mathrm{~km}$ & $603.4 \mathrm{~km}$ & $126 \mathrm{~m}$ \\
\hline
\end{tabular}

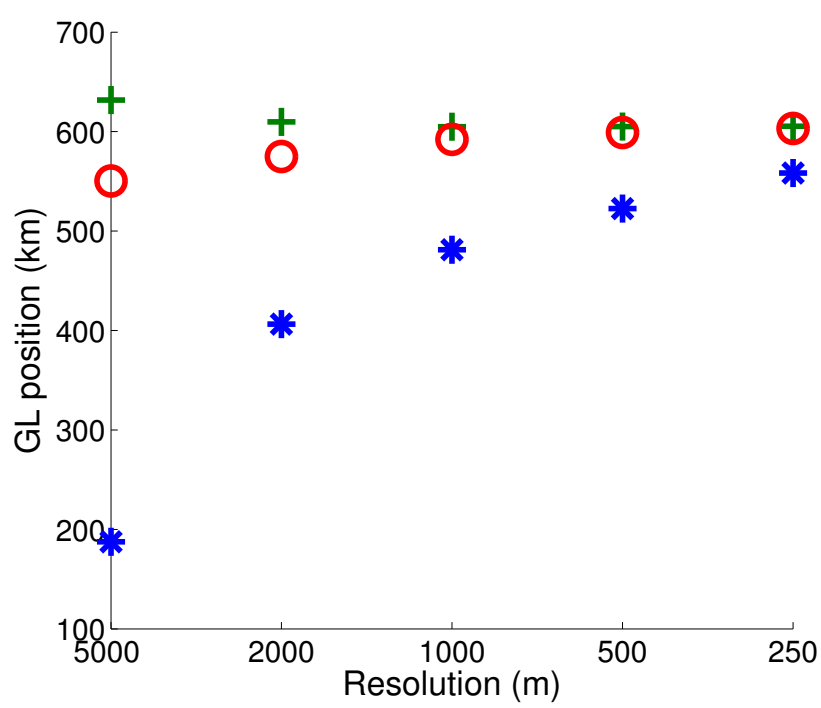

Figure 2. Steady state grounding line position in $y=0$ as a function of mesh refinement for NSEP (blue stars), SEP1 (green crosses) and SEP2 (red circles).

from $5 \mathrm{~km}$ to $250 \mathrm{~m}$, for a number of elements varying between 2553 and 1013894 depending on the spatial resolution. The first three grounding line parameterizations (NSEP, SEP1 and SEP2) are run for all mesh resolutions, resulting in a total of 15 simulations. The last grounding line parameterization (SEP3) is only run to find the initial steady state grounding line position for meshes of $5 \mathrm{~km}$ and $1 \mathrm{~km}$ resolution, with a varying number of integration points (19 simulations for each mesh resolution).

\section{Results}

We consider that steady state is reached when the rate of change in ice thickness, grounding line position and ice velocity are all respectively lower than $10^{-5} \mathrm{~m} \mathrm{yr}^{-1}$, $10^{-3} \mathrm{~m} \mathrm{yr}^{-1}$ and $10^{-5} \mathrm{~m} \mathrm{yr}^{-2}$, respectively. It takes approximately 50000 years to reach steady state. We need to ensure the Courant-Friedrichs-Lewy condition (CFL, Courant et al., 1967) for all models, so meshes with finer resolution require smaller time steps than the ones with coarser resolution. The initial grounding line position for each of the NSEP, SEP1 and SEP2 models is summarized in Table 1. It varies between $x=188 \mathrm{~km}$ and $x=632 \mathrm{~km}$ depending on the model resolution and grounding line parameterization. In the case of NSEP, the grounding position varies by several hundreds of kilometers (between 188 and $558 \mathrm{~km}$ ), while SEP1 and SEP2 lead to variations in steady state grounding line positions of $50 \mathrm{~km}$ or less (between 605 and $632 \mathrm{~km}$ and between 550 and $603 \mathrm{~km}$, respectively for the SEP1 and SEP2). This spread in grounding line positions is larger than in Feldmann et al. (2014). Steady state grounding line positions at $y=0 \mathrm{~km}$ for these three parameterizations and all mesh resolutions are shown on Fig. 2. Grounding line is moving upstream as the mesh resolution increases for SEP1, while it is moving downstream for NSEP and SEP2. Steadystate grounding line positions found with SEP3 are in good agreement with SEP2 for both 5 and $1 \mathrm{~km}$ mesh resolutions. It varies between $x=540$ and $x=497 \mathrm{~km}$, and $x=584$ and $x=589 \mathrm{~km}$ for mesh resolutions of 5 and $1 \mathrm{~km}$, depending on the integration order (see Fig. 5), which is respectively within 10 and $3 \mathrm{~km}$ of SEP2 for a similar resolution when using enough integration points.

As for the domain configuration, the model parameterization and forcings do not vary in the $y$ direction and we have 
$u_{y}(x, 0)=u_{y}(x, 50)=0$, the grounding line position should therefore be a straight line parallel to the $y$ axis. In practice, this position slightly varies with $y$, especially since we use an unstructured mesh. We define the grounding line span as follows:

$\delta \mathrm{GL}=\max \left(x_{\mathrm{gi}}\right)-\min \left(x_{\mathrm{gi}}\right)$,

where $x_{\mathrm{gi}}$ are all grounding line positions for $0<y<50 \mathrm{~km}$. The grounding line span is presented in Table 1 and provides a quantification of the spread of grounding line positions. $\delta \mathrm{GL}$ is about twice the size of the elements for NSEP and less than half this size for SEP1 and SEP2.

The perturbation experiment is performed to analyze the reversibility of the grounding line position in a simplified configuration. Figure 3 shows that grounding line advances along the glacier centerline as the basal friction is reduced in this area, and retreats along the free slip boundary. Advance and retreat extents vary depending on grounding line parameterizations and mesh resolutions. Distances of advance along the centerline and retreat along the free-slip boundary after 100 years for all 15 simulations are presented in Table 2 . Advances are more pronounced and retreats are reduced at low resolutions, except for SEP1 that exhibits similar advance and retreat for all mesh sizes. Both SEP1 and SEP2 present advance and retreat after 100 years that converged toward 10 and $6.5 \mathrm{~km}$ respectively at high resolution.

The updated steady state position reached after the perturbation experiment is identical to the initial steady state position (Fig. 3), except for NSEP simulations at low resolution (more than $1 \mathrm{~km}$ resolution), so most simulations exhibit reversibility. The difference between the initial and final grounding line position is less than $10 \mathrm{~m}$ in all the cases where the two steady state grounding line positions superimpose on Fig. 3.

To analyze the motion of the grounding line during the perturbation experiment, Fig. 4 presents the 100 year advance and first 100 year retreat of the grounding line position during the basal perturbation experiment for the different resolutions and grounding line parameterizations. Migration of grounding line position for $y=0$ and $y=50 \mathrm{~km}$ is shown (one value every year). For NSEP (first column), grounding line position advances and retreats in discrete steps that are linked to the element size. For both SEP1 and SEP2 (second and third columns), the advance and retreat are continuous. Grounding line advance at $y=50 \mathrm{~km}$ takes between 20 and 40 years to reach its most advanced position. In the case of NSEP, the grounding position remains stable after the advance, while for SEP1, SEP2 and NSEP at $250 \mathrm{~m}$ resolution, it is followed by a small retreat. Grounding line retreat at $y=0 \mathrm{~km}$ takes longer than the advance at $y=50 \mathrm{~km}$ and is still evolving after 100 years in most cases, which shows that the grounding line is still far from having reached a new steady state position.
Table 2. Grounding line displacement during the perturbation experiment for the 15 simulations.

\begin{tabular}{llrr}
\hline GL parameterization & Resolution & $\begin{array}{r}\Delta \mathrm{GL} \\
(y=0 \mathrm{~km})\end{array}$ & $\begin{array}{r}\Delta \mathrm{GL} \\
(y=50 \mathrm{~km})\end{array}$ \\
\hline NSEP & $5 \mathrm{~km}$ & $31.3 \mathrm{~km}$ & $-12.5 \mathrm{~km}$ \\
NSEP & $2 \mathrm{~km}$ & $18.8 \mathrm{~km}$ & $-2.1 \mathrm{~km}$ \\
NSEP & $1 \mathrm{~km}$ & $18.7 \mathrm{~km}$ & $-2.0 \mathrm{~km}$ \\
NSEP & $500 \mathrm{~m}$ & $15.6 \mathrm{~km}$ & $-2.6 \mathrm{~km}$ \\
NSEP & $250 \mathrm{~m}$ & $13.1 \mathrm{~km}$ & $-4.8 \mathrm{~km}$ \\
\hline SEP1 & $5 \mathrm{~km}$ & $9.6 \mathrm{~km}$ & $-7.1 \mathrm{~km}$ \\
SEP1 & $2 \mathrm{~km}$ & $10.0 \mathrm{~km}$ & $-6.9 \mathrm{~km}$ \\
SEP1 & $1 \mathrm{~km}$ & $9.8 \mathrm{~km}$ & $-6.5 \mathrm{~km}$ \\
SEP1 & $500 \mathrm{~m}$ & $10.0 \mathrm{~km}$ & $-6.4 \mathrm{~km}$ \\
SEP1 & $250 \mathrm{~m}$ & $10.1 \mathrm{~km}$ & $-6.4 \mathrm{~km}$ \\
\hline SEP2 & $5 \mathrm{~km}$ & $15.1 \mathrm{~km}$ & $-4.1 \mathrm{~km}$ \\
SEP2 & $2 \mathrm{~km}$ & $12.1 \mathrm{~km}$ & $-5.0 \mathrm{~km}$ \\
SEP2 & $1 \mathrm{~km}$ & $10.4 \mathrm{~km}$ & $-6.1 \mathrm{~km}$ \\
SEP2 & $500 \mathrm{~m}$ & $10.6 \mathrm{~km}$ & $-6.2 \mathrm{~km}$ \\
SEP2 & $250 \mathrm{~m}$ & $10.4 \mathrm{~km}$ & $-6.3 \mathrm{~km}$ \\
\hline
\end{tabular}

\section{Discussion}

In this study, we investigate the influence of grounding line parameterization on grounding line steady state position as well as its dynamic response to a perturbation in basal friction in the grounding line area. All grounding line parameterizations show a dependence on mesh resolution as well as convergence of the grounding line steady state position with finer mesh resolution. Convergence of grounding line steady state position is achieved within a few kilometers for SEP1 and SEP2, while it has not fully converged for NSEP. Even at $250 \mathrm{~m}$ resolution, grounding line position using NSEP is located several tens of kilometers upstream of SEP1 and SEP2 grounding line position. This behavior is also observed for the HSE model in Pattyn et al. (2013) that was also relying on ISSM and did not include sub-element parameterization of grounding line position. The mesh resolution of this model around the grounding line was $200 \mathrm{~m}$ and the grounding line steady state position is located at $545 \mathrm{~km}$, which is about $50 \mathrm{~km}$ upstream of the other SSA models and consistent with the results presented here. Indeed, in the case of NSEP, the grounding line is located at the last grounded point. Basal friction downstream of this point is set to zero so the resistance from basal friction is reduced, and ice flows faster. This leads to a thinner ice sheet and a simulated grounding line position upstream of the one computed with models that include sub-element parameterization. It is a coincidence that this position is similar to models that include vertical shear, which reduces the effective ice viscosity and also results in faster flow and grounding line position farther upstream (Pattyn et al., 2013). Results of the FPA2 model, also performed with an SSA model, NSEP and the same $200 \mathrm{~m}$ resolution 

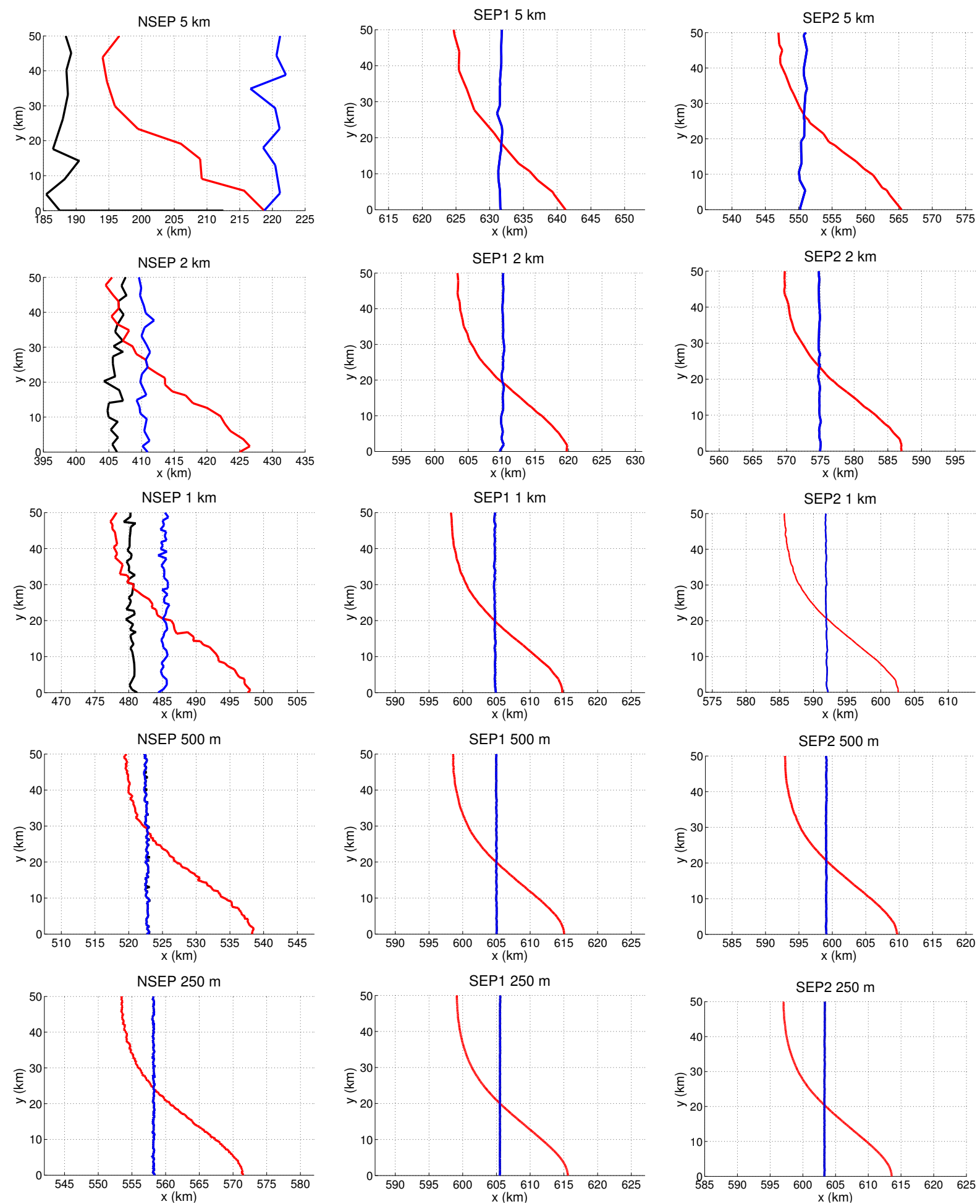

Figure 3. Initial steady state grounding line positions in the $(x, y)$ plane (black line), position 100 years after the basal perturbation is introduced (red line) and new steady state position after the basal friction is reset to its initial value (blue line). Where black line is not visible, black and blue lines superimpose. $x$ and $y$ axis have the same scale for all plots. 

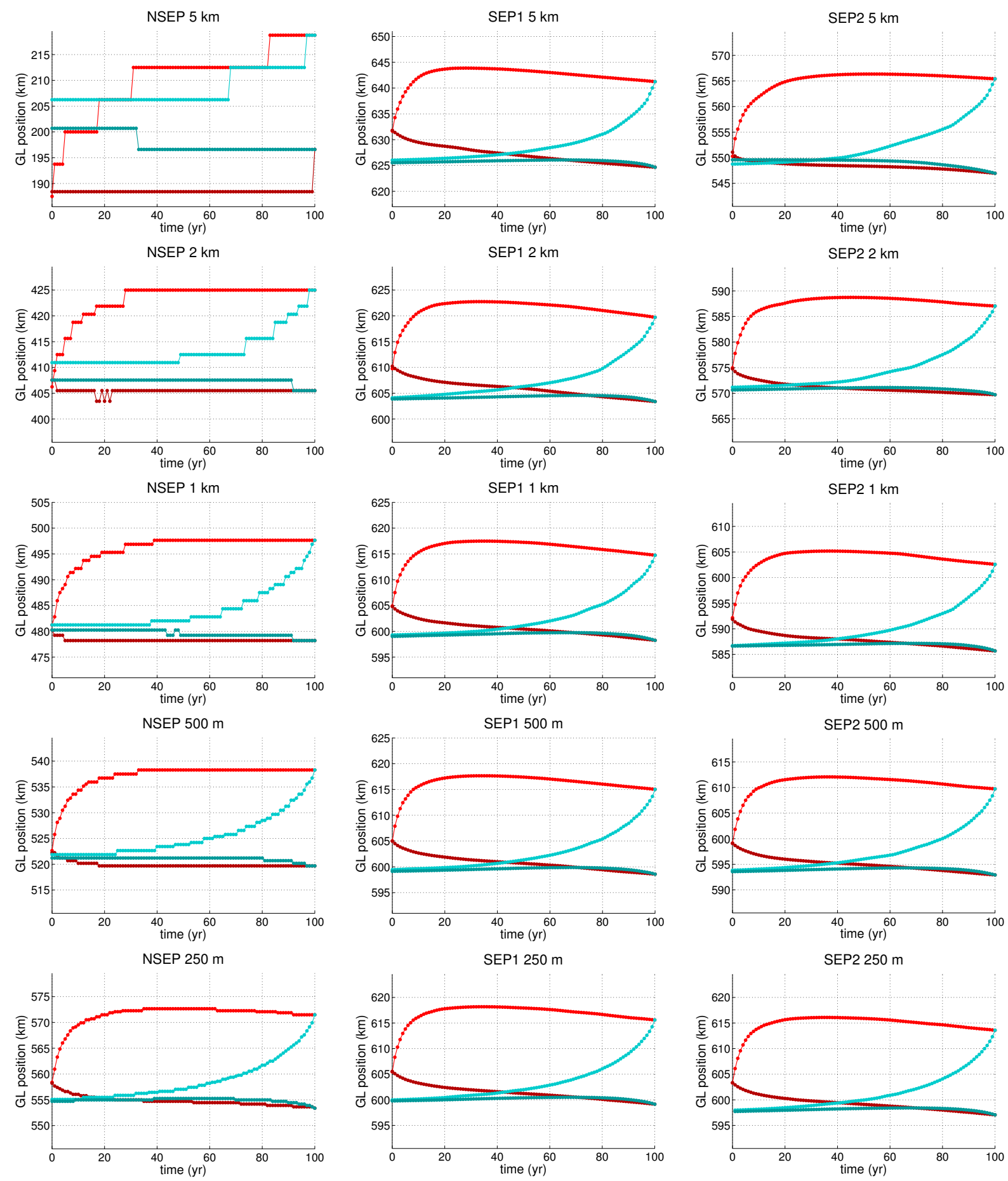

Figure 4. Time-dependent position of the grounding line along the symmetry axis $(y=0)$ and the free slip border $(y=50)$ during (respectively light red and dark red) and after (respectively light teal and dark teal) the friction perturbation for coarse mesh resolutions. $y$ axes have the same scale for all simulations. $x$ axes (time) is reversed after the perturbation experiment (teal lines).

shows a similar behavior to a smaller extent, with a grounding line position located around $580 \mathrm{~km}$.

The results presented here show that proper grounding line parameterization is crucial for marine ice sheet simulations as discrepancies of several tens of kilometers exist between the different parameterizations and sub-element parameterization should be included. The steady state grounding line positions using SEP1 and SEP2 are consistent with models presented in Pattyn et al. (2013). Differences between simulations carried out with and without SEP are as large as those 
performed with different stress balance approximations in Pattyn et al. (2013), demonstrating the critical impact of SEP. For example at $500 \mathrm{~m}$ resolution, the steady-state grounding line position varies between 522 and $605 \mathrm{~km}$ for NSEP and SEP1 respectively, so more than $80 \mathrm{~km}$. In Pattyn et al. (2013), the same grounding line position computed with FS and hybrid L1L2 models (Hindmarsh, 2004) varies by less than $10 \mathrm{~km}$, and by up to $80 \mathrm{~km}$ between FS and SSA models.

Some previous results on flow-band models (Gladstone et al., 2010a) exhibit unstable behavior in grounding line retreat in the case of NSEP. We did not experience this kind of behavior and all simulations were stable and converged to a steady state position. Grounding line advance and retreat was also continuous and located anywhere within the element, with no sign of preferred position within the element as observed in Gladstone et al. (2010a) when using SEP1 and SEP2. The second horizontal dimension of our model and the unstructured nature of our mesh may explain these differences.

As expected, grounding line span, $\delta \mathrm{GL}$, is higher than model resolution for the NSEP while it is less than half of the model resolution for SEP1 and SEP2 (see Table 1). Differences in grounding line position between models based on a 500 and $250 \mathrm{~m}$ mesh resolution is respectively $25,0.5$ and $3.2 \mathrm{~km}$ for NSEP, SEP1 and SEP2. This suggests that grounding line position has not converged for NSEP, while the convergence error is 0.5 and $3.2 \mathrm{~km}$ respectively for the SEP1 and SEP2, as defined in Gladstone et al. (2010a, b).

In the reversibility test, all models except NSEP at a resolution equal or higher than $1 \mathrm{~km}$ satisfy the reversibility condition. Numerical requirement to satisfy the reversibility criterion is therefore a resolution below $1 \mathrm{~km}$ for NSEP; whereas all models based on sub-element parameterization exhibit reversibility even when relying on a coarse mesh. These results are consistent with Feldmann et al. (2014): reversibility is observed for grid resolutions lower than $2 \mathrm{~km}$ for NSEP and with grid resolutions as low as $16 \mathrm{~km}$ when SEP is applied. The reversibility criterion is a however necessary condition that provides insights in the numerical aspects of the marine ice sheet model and the simulations, but this test can be passed at relatively low resolutions for which steady-state grounding line positions are not accurate. It therefore does not guarantee the accuracy of the numerical treatment of the grounding line and sufficient mesh resolution, as suggested by the large number of our simulations that verify the reversibility with different steady state grounding line positions.

If we compare SEP1 and SEP2, Fig. 2 shows that they both converge towards the same position for fine mesh resolutions, but that positions at coarser resolutions are upstream of the "converged" position for SEP1, and downstream for SEP2. The dynamic advance is also slightly different: grounding line advance at $y=0 \mathrm{~km}$ is faster and goes farther for the SEP1. It is also associated to a larger retrograde retreat in the second part of the experiment, which is especially pronounced at low resolutions. The grounding line retreat at $y=50 \mathrm{~km}$ is also larger for SEP1 at low resolution, but both exhibit similar behaviors for resolutions finer than $2 \mathrm{~km}$. A mesh resolution finer than $2 \mathrm{~km}$ should therefore be employed to accurately capture dynamic behavior or marine ice sheet in this configuration. SEP2 is a more "exact" solution, as basal friction is integrated over the exact grounded part of the element, while SEP1 uses an area scaling of the basal friction. In this experiment, the basal friction is uniform over the whole domain, so it is not surprising that SEP1 and SEP2 lead to similar results. We expect greater differences to appear in the case where basal friction varies over the domain, but this is beyond the scope of this paper.

SEP3 was tested only to find the steady-state position of the grounding line on the 5 and $1 \mathrm{~km}$ meshes. This method only allows a finite number of grounding line positions to be captured within the element contrary to the other two SEP. We tested this solution with integration orders going from 2 to 20. Results in Fig. 5 show that increasing the number of Gauss points only have an impact on grounding line position for coarse mesh resolutions. For the $1 \mathrm{~km}$ mesh, integration with order of 4 or below leads to one position, and integration with order of 5 and above leads to a second position; however, the grounding line position is within $3 \mathrm{~km}$ of the SEP2. For the $5 \mathrm{~km}$ mesh, the spread in grounding line positions is much larger, with steady-state grounding line positions varying by more than $50 \mathrm{~km}$. If the integration order is greater than 12 , however, these positions is located within $10 \mathrm{~km}$ of the SEP2 position. Increasing the number of integration points is therefore a simple solution to include basal friction in a portion of the element in a finite element framework, and provides results similar to other sub-element parameterizations if the integration order is sufficient. This method should be further investigated using a larger range of mesh resolutions to ensure convergence of the grounding line position at finer mesh resolutions.

The results presented in this paper were all performed using a 2-D SSA model and unstructured uniform isotropic meshes. Refinement away from the grounding line is important to accurately capture shear margins (Raymond, 1996) or topography that varies over short distances, but should not be uniform and be based, for example, on the Hessian of the velocity (Morlighem et al., 2010). Increasing mesh resolution has a double impact on computational time. First, increasing the number of degrees of freedom increases computational time, mainly when solving the linear systems. Second, as the elements are smaller, the time steps allowed in transient simulations in order to fulfill the CFL condition are reduced. Fine mesh resolution is therefore necessary in critical areas but alternatives less computationally intensive should also be explored. Adding grounding line parameterizations is a simple improvement as grounding line positions are better captured at no additional cost. Sub-element parameterization allows grounding line position to be anywhere within an element, but the shape of the grounding line is still constrained 

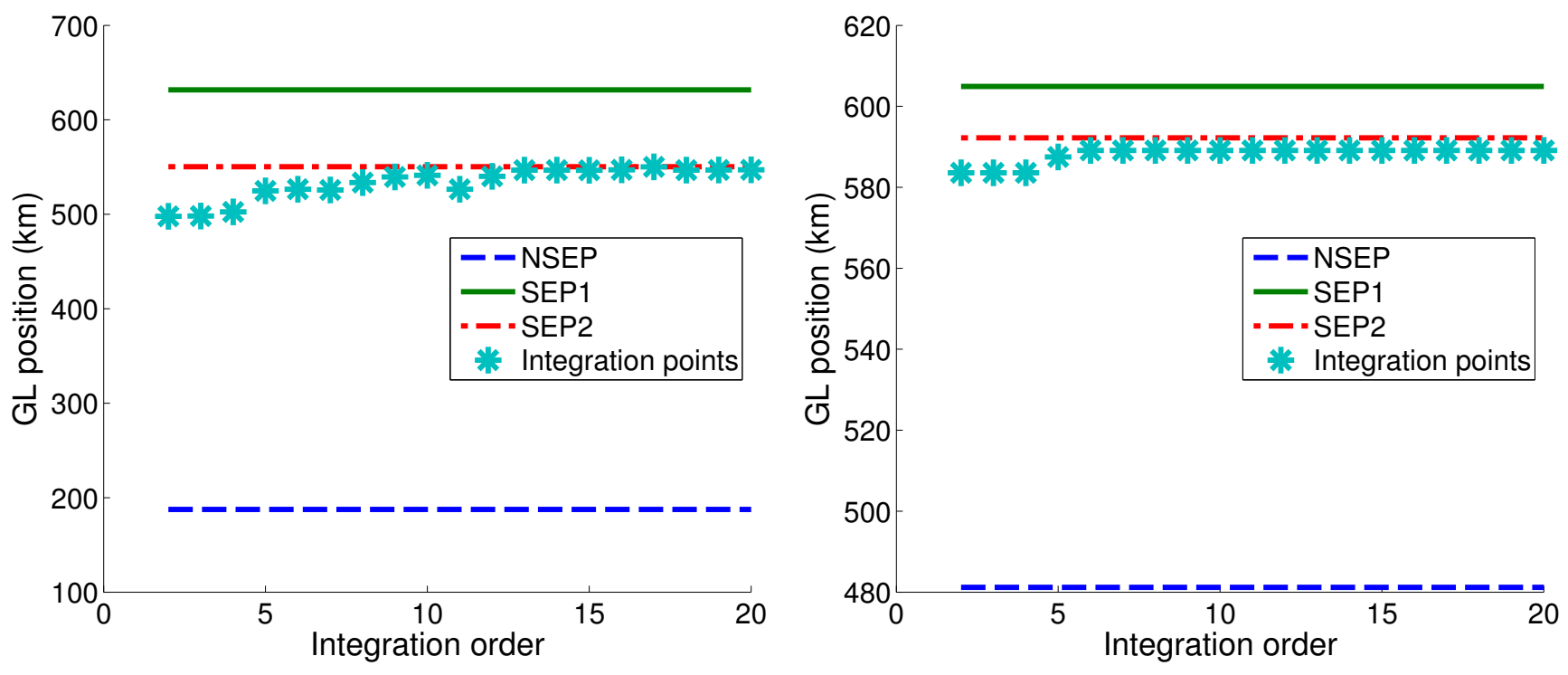

Figure 5. Grounding line position in $y=0$ for $5 \mathrm{~km}$ (left panel) and $1 \mathrm{~km}$ (right panel) resolution mesh for NSEP (dark-blue dashed line), SEP1 (green straight line), SEP2 (red dash dotted line) and SEP3 with different integration orders (light blue stars).

by the mesh resolution: exact grounding line position within an element remains a straight line if piecewise linear elements are used. Mesh refinement and parameterizations are therefore two methods that should be combined.

This study shows that different grounding line parameterizations lead to different grounding line steady state positions as well as different dynamic behaviors. Differences in model simulations performed with and without SEP are as large as differences between models relying on different ice flow approximations in the MISMIP3D results (Pattyn et al., 2013), which demonstrate the importance of grounding line parameterization. We expect our results to be similar for higher-order (HO) models (Blatter, 1995; Pattyn, 2003). This is because HO models are similar to SSA (HO models include vertical shear stress as well), and the grounding line position is based on the hydrostatic condition in both cases. Models that do not include sub-element parameterizations will need a significantly finer mesh resolution to converge, and the grounding line position may likely be located further upstream than those based on a sub-element parameterization. Recent studies show that relying on full-Stokes in some critical areas in the model domain is necessary (Hindmarsh, 2004; Gudmundsson, 2008; Morlighem et al., 2010), and that grounding line position is better resolved using a contact mechanics condition in this case (Nowicki and Wingham, 2008; Durand et al., 2009b). This condition, however, is only evaluated on the edge or face on which the stress tensor is computed, and no SEP has yet been formulated for such models. This may explain why a very fine resolution on the order of tens of meters must be employed to model grounding line dynamics with FS in some cases (Durand et al., 2009b).

\section{Conclusions}

In this study, we used ISSM with a two-dimensional shelfystream approximation with fixed unstructured meshes of varying resolution and the MISMIP3D set-up to investigate the impact of several grounding line parameterizations on grounding line dynamics. We show that mesh refinement and grounding line parameterization both have a significant influence on modeled grounding line positions, as well as advance and retreat rates. Models that do not use sub-element parameterizations of grounding line position exhibit a steady state grounding line position located at least several tens of kilometers further upstream than those computed with subelement parameterizations, even at a high spatial resolution of $250 \mathrm{~m}$. Differences between simulations performed with and without sub-element parameterization are as large as those performed with different approximations of the stress balance in this configuration and the reversibility criterion is satisfied at a much coarser resolution that the one required to reach convergence for the steady-state grounding line position. We therefore do not recommend using fixed mesh models that do not rely on sub-element parameterization unless sensitivity to mesh resolution is thoroughly tested. All the sub-element parameterizations tested converged towards the same results at high resolution, and we suggest that mesh refinement in grounding line areas should remain below about $2 \mathrm{~km}$, as results with different sub-parameterizations are all similar at these resolutions in the simulations presented here. 


\section{Appendix A: Description of basal friction integration}

We detail here the stiffness matrices associated to basal friction on grounded ice for the different sub-element parameterizations. Let $\mathcal{V}$ be the space of kinematically admissible velocity fields and $\Phi=\left(\phi_{x}, \phi_{y}\right) \in \mathcal{V}$ a kinematically admissible velocity field. For any $\Phi \in \mathcal{V}$ the stiffness matrix in the case of NSEP is

$\mathbf{K}_{\mathrm{f}}=\int_{\Gamma_{\mathrm{g}}} C \boldsymbol{u}_{\mathrm{b}} \cdot \Phi \mathrm{d} \Gamma$,

where $\Gamma_{\mathrm{b}}$ is the lower surface of the ice sheet where ice is grounded.

Using a decomposition over the elements and using integration points to calculate the integral gives

$\mathbf{K}_{\mathrm{f}}=\sum_{E_{\mathrm{g}}} \sum_{g} C \boldsymbol{u}_{\mathrm{b}}(g) \cdot \Phi(g) W_{\mathrm{g}}$,

where $E_{\mathrm{g}}$ are the grounded elements, $g$ the integration points used for the integration and $W_{\mathrm{g}}$ the weight associated to each integration point.

For SEP1, the friction coefficient is affected by the grounded area of each element, so the stiffness matrix is

$\mathbf{K}_{\mathrm{f}}=\sum_{E_{\mathrm{g}}} \sum_{g} C_{\mathrm{g}} \boldsymbol{u}_{\mathrm{b}}(g) \cdot \Phi(g) W_{\mathrm{g}}$, where $C_{\mathrm{g}}$, Eq. (7), is the applied basal friction coefficient for elements partially grounded $\left(C_{\mathrm{g}}=C\right.$ for elements completely grounded).

For SEP2, the friction is applies only on the grounded part of the element, so the domain of integration is changed to $\widetilde{E}_{\mathrm{g}}$ instead of $E_{\mathrm{g}}$ :

$\mathbf{K}_{\mathrm{f}}=\sum_{\widetilde{E}_{\mathrm{g}}} \sum_{g} C \boldsymbol{u}_{\mathrm{b}}(g) \cdot \Phi t(g) W_{\mathrm{g}}$,

where $\widetilde{E}_{\mathrm{g}}$ corresponds exactly to the brown area on Fig. 1d. In the code, this is done by creating sub-regions within each element partly grounded by determining the exact location of the points where $H=H_{\mathrm{f}}$ and changing the integration domain over these sub-regions.

For SEP3, the stiffness matrix is changed to

$\mathbf{K}_{\mathrm{f}}=\sum_{E_{\mathrm{g}}} \sum_{g} C \delta(g) \boldsymbol{u}_{\mathrm{b}}(g) \cdot \Phi(g) W_{\mathrm{g}}$,

where $\delta(g)$ is evaluated at each integration point:

$\delta(g)=\left\{\begin{array}{lll}1 & \text { if } \quad H>H_{\mathrm{f}} \\ 0 & \text { if } \quad H \leq H_{\mathrm{f}}\end{array}\right.$. 
Acknowledgements. H. Seroussi was supported by an appointment to the NASA Postdoctoral Program at the Jet Propulsion Laboratory, administered by Oak Ridge Associated Universities through a contract with NASA. This work was performed at the Jet Propulsion Laboratory, California Institute of Technology, and at the Department of Earth System Science, University of California Irvine. A. Khazendar and M. Morlighem were supported by grants from the National Aeronautics and Space Administration's Cryospheric Sciences Program. E. Larour was supported by grants from NASA's Cryospheric Sciences and Modeling, Analysis and Prediction Programs. We thank R. Gladstone, F. Pattyn, G. Durand and A. Levermann for their suggestions which improved the quality of the manuscript.

Edited by: O. Gagliardini

\section{References}

Blatter, H.: Velocity And Stress-Fields In Grounded Glaciers: A Simple Algorithm For Including Deviatoric Stress Gradients, J. Glaciol., 41, 333-344, 1995.

Bohlander, J. and Scambos, T.: Antarctic coastlines and grounding line derived from MODIS Mosaic of Antarctica (MOA), digital media, Natl. Snow and Ice Data Cent., Boulder, 2007.

Cornford, S., Martin, D., Graves, D., Ranken, D. F., Le Brocq, A. M., Gladstone, R., Payne, A., Ng, E., and Lipscomb, W.: Adaptive mesh, finite volume modeling of marine ice sheets, J. Comput. Phys., 232, 529-549, doi:10.1016/j.jcp.2012.08.037, 2013

Courant, R., Friedric, K., and Lewy, H.: On Partial Difference Equations Of Mathematical Physics, Ibm J. Res. Develop., 11, 215-234, 1967.

Cuffey, K. and Paterson, W. S. B.: The Physics of Glaciers, 4th Edn., Elsevier, Oxford, 2010.

Durand, G., Gagliardini, O., de Fleurian, B., Zwinger, T., and Le Meur, E.: Marine ice sheet dynamics: Hysteresis and neutral equilibrium, J. Geophys. Res., 114, 1-10, doi:10.1029/2008JF001170, 2009a.

Durand, G., Gagliardini, O., Zwinger, T., Le Meur, E., and Hindmarsh, R.: Full Stokes modeling of marine ice sheets: influence of the grid size, Ann. Glaciol., 50, 109-114, $2009 \mathrm{~b}$.

Favier, L., Durand, G., Cornford, S. L., Gudmundsson, G. H., Gagliardini, O., Gillet-Chaulet, F., Zwinger, T., Payne, A. J., and Le Brocq, A.: Retreat of Pine Island Glacier controlled by marine ice-sheet instability, Nat. Clim. Change, 4, 117-121, doi:10.1038/NCLIMATE2094, 2014.

Feldmann, J., Albrecht, T., Khroulev, C., F., P., and Levermann, A.: Resolution-dependent performance of grounding line motion in a shallow model compared with a full-Stokes model according to the MISMIP3d intercomparison, J. Glaciol., 60, 353-359, doi:10.3189/2014JoG13J093, 2014.

Gladstone, R. M., Lee, V., Vieli, A., and Payne, A. J.: Grounding line migration in an adaptive mesh ice sheet model, J. Geophys. Res., 115, 1-19, doi:10.1029/2009JF001615, 2010a.

Gladstone, R. M., Payne, A. J., and Cornford, S. L.: Parameterising the grounding line in flow-line ice sheet models, The Cryosphere, 4, 605-619, doi:10.5194/tc-4-605-2010, 2010 b.
Goldberg, D., Holland, D. M., and Schoof, C.: Grounding line movement and ice shelf buttressing in marine ice sheets, J. Geophys. Res., 114, 1-23, doi:10.1029/2008JF001227, 2009.

Goldstein, R., Engelhardt, H., Kamb, B., and Frolich, R.: Satellite Radar Interferometry for Monitoring ice-sheet motion: Application to an antarctic ice stream, Science, 262, 1525-1530, 1993.

Gudmundsson, G. H.: Analytical solutions for the surface response to small amplitude perturbations in boundary data in the shallow-ice-stream approximation, The Cryosphere, 2, 77-93, doi:10.5194/tc-2-77-2008, 2008.

Hindmarsh, R.: A numerical comparison of approximations to the Stokes equations used in ice sheet and glacier modeling, J. Geophys. Res., 109, 1-15, doi:10.1029/2003JF000065, 2004.

Hindmarsh, R. and Le Meur, E.: Dynamical processes involved in the retreat of marine ice sheets, J. Glaciol., 47, 271-282, 2001.

Huybrechts, P.: A 3-D model for the Antarctic ice sheet: a sensitivity study on the glacial-interglacial contrast, Clim. Dynam., 5, 79-92, 1990.

Katz, R. F. and Worster, M.: Stability of ice-sheet grounding lines, P. Roy. Soc. A, 466, 1597-1620, doi:10.1098/rspa.2009.0434, 2010.

Larour, E., Seroussi, H., Morlighem, M., and Rignot, E.: Continental scale, high order, high spatial resolution, ice sheet modeling using the Ice Sheet System Model (ISSM), J. Geophys. Res., 117, 1-20, doi:10.1029/2011JF002140, 2012.

MacAyeal, D.: Large-scale ice flow over a viscous basal sediment: Theory and application to Ice Stream B, Antarctica, J. Geophys. Res., 94, 4071-4087, 1989.

Morlighem, M., Rignot, E., Seroussi, H., Larour, E., Ben Dhia, H., and Aubry, D.: Spatial patterns of basal drag inferred using control methods from a full-Stokes and simpler models for Pine Island Glacier, West Antarctica, Geophys. Res. Lett., 37, 1-6, doi:10.1029/2010GL043853, 2010.

Nowicki, S. M. J. and Wingham, D. J.: Conditions for a steady ice sheet-ice shelf junction, Earth Planet. Sc. Lett., 265, 246-255, 2008.

Pattyn, F.: A new three-dimensional higher-order thermomechanical ice sheet model: Basic sensitivity, ice stream development, and ice flow across subglacial lakes, J. Geophys. Res., 108, 1-15, doi:10.1029/2002JB002329, 2003.

Pattyn, F. and Durand, G.: Why marine ice sheet model predictions may diverge in estimating future sea level rise, Geophys. Res. Lett., 40, 4316-4320, doi:10.1002/grl.50824, 2013.

Pattyn, F., Huyghe, A., De Brabander, S., and De Smedt, B.: Role of transition zones in marine ice sheet dynamics, J. Geophys. Res.Earth, 111, 1-10, doi:10.1029/2005JF000394, 2006.

Pattyn, F., Schoof, C., Perichon, L., Hindmarsh, R. C. A., Bueler, E., de Fleurian, B., Durand, G., Gagliardini, O., Gladstone, R., Goldberg, D., Gudmundsson, G. H., Huybrechts, P., Lee, V., Nick, F. M., Payne, A. J., Pollard, D., Rybak, O., Saito, F., and Vieli, A.: Results of the Marine Ice Sheet Model Intercomparison Project, MISMIP, The Cryosphere, 6, 573-588, doi:10.5194/tc6-573-2012, 2012.

Pattyn, F., Perichon, L., Durand, G., Favier, L., Gagliardini, O., Hindmarsh, R. C. A., Zwinger, T., Albrecht, T., Cornford, S., Docquier, D., Fuerst, J., Goldberg, D., Gudmundsson, H., Humbert, A., Hutten, M., Huybrecht, P., Jouvet, G., Kleiner, T., Larour, E., Martin, D., Morlighem, M., Payne, A., Pollard, D., Ruckamp, M., Rybak, O., Seroussi, H., Thoma, M., and 
Wilkens, N.: Grounding-line migration in plan-view marine icesheet models: results of the ice2sea MISMIP3d intercomparison, J. Glaciol., 59, 410-422, doi:10.3189/2013JoG12J129, 2013.

Raymond, C.: Shear margins in glaciers and ice sheets, J. Glaciol., 42, 90-102, 1996.

Rignot, E., Mouginot, J., and Scheuchl, B.: Antarctic grounding line mapping from differential satellite radar interferometry, Geophys. Res. Lett., 38, 1-6, doi:10.1029/2011GL047109, 2011 a.

Rignot, E., Velicogna, I., van den Broeke, M., Monaghan, A., and Lenaerts, J.: Acceleration of the contribution of the Greenland and Antarctic ice sheets to sea level rise, Geophys. Res. Lett., 38, 1-5, doi:10.1029/2011GL046583, 2011b.

Ritz, C., Rommelaere, V., and Dumas, C.: Modeling the evolution of Antarctic ice sheet over the last 420,000 years: Implications for altitude changes in the Vostok region, J. Geophys. Res., 106, 31943-31964, doi:10.1029/2001JD900232, 2001.

Schodlok, M., Menemenlis, D., Rignot, E., and Studinger, M.: Sensitivity of the ice-shelf/ocean system to the sub-ice-shelf cavity shape measured by NASA IceBridge in Pine Island Glacier, West Antarctica, Ann. Glaciol., 53, 156-162, doi:10.3189/2012AoG60A073, 2012.
Schoof, C.: Marine ice-sheet dynamics. Part 1. The case of rapid sliding, J. Fluid Mech., 573, 27-55, doi:10.1017/S0022112006003570, 2007a.

Schoof, C.: Ice sheet grounding line dynamics: Steady states, stability, and hysteresis, J. Geophys. Res., 112, 1-19, doi:10.1029/2006JF000664, 2007b.

van der Veen, C.: Response of a Marine Ice-Sheet to Changes at the Grounding Line, Quaternary Res., 24, 257-267, doi:10.1016/0033-5894(85)90049-3, 1985.

Vieli, A. and Payne, A.: Assessing the ability of numerical ice sheet models to simulate grounding line migration, J. Geophys. Res., 110, 1-18, doi:10.1029/2004JF000202, 2005.

Weertman, J.: Stability of the junction of an ice sheet and an ice shelf, J. Glaciol., 13, 3-11, 1974.

Winkelmann, R., Martin, M. A., Haseloff, M., Albrecht, T., Bueler, E., Khroulev, C., and Levermann, A.: The Potsdam Parallel Ice Sheet Model (PISM-PIK) - Part 1: Model description, The Cryosphere, 5, 715-726, doi:10.5194/tc-5-715-2011, 2011.

Zienkiewicz, O. C. and Taylor, R. L.: The finite element method, vol. 1, 4th Edn., New York, London, 1989. 